\title{
Torque motor tape winding characteristics and its connection with design parameters
}

\author{
Antonina Dolgih", and Vladimir Martemyanov \\ Tomsk Polytechnic University, 634050 Tomsk, Russia
}

\begin{abstract}
The paper considers the features of a tape winding torque motor development. Novel motor type allows to obtain an increased torque by the high current flowing through the tape winding. It is shown that the most effective way of the torque increasing is the tape winding plate resistance reducing.
\end{abstract}

\section{Introduction}

At present, torque motors are used in technical systems as the gearless drive base. The torque motor has a modular structure, consists of two separate assembly units: a rotor and a stator. The modular motor structure is used for direct integration into the mechanism. The rotor contains high-coercive rare-earth magnets. The stator has two design versions: "a smooth stator" and "a grooving stator". For precise systems "the smooth stator" is preferable, because there is no cogging torque.

The current state of the torque motors development is performed in [1-4]. The analysis shows that the specific characteristics of Russian torque motors are worse than foreign analogues. Torque motors can be improved by using new magnetic materials, changing the rotor design or using new types of windings. In our opinion, the most promising way is the third approach. In this case the specific characteristics increasing can be achieved by the torque raising due to the high current flowing through the rotor winding. In this case, there are some problems with the motor thermal mode, because the power consumption is mainly released as heat in the windings.

In this situation, it is proposed to replace the traditional winding of the engine with a tape winding with side cutouts. The heat in the winding will be directly transferred to the motor case, and the developed torque increasing will occur due to the high current flowing through the tape winding. In addition, a new motor stator design and technological approach are proposed, including the use of additive technologies; and the possibility of creating the necessary "torque-rotor angle" characteristic. Figure 1 shows the scheme of the tape winding torque motor.

\footnotetext{
* Corresponding author: ivanovatonya@tpu.ru
} 


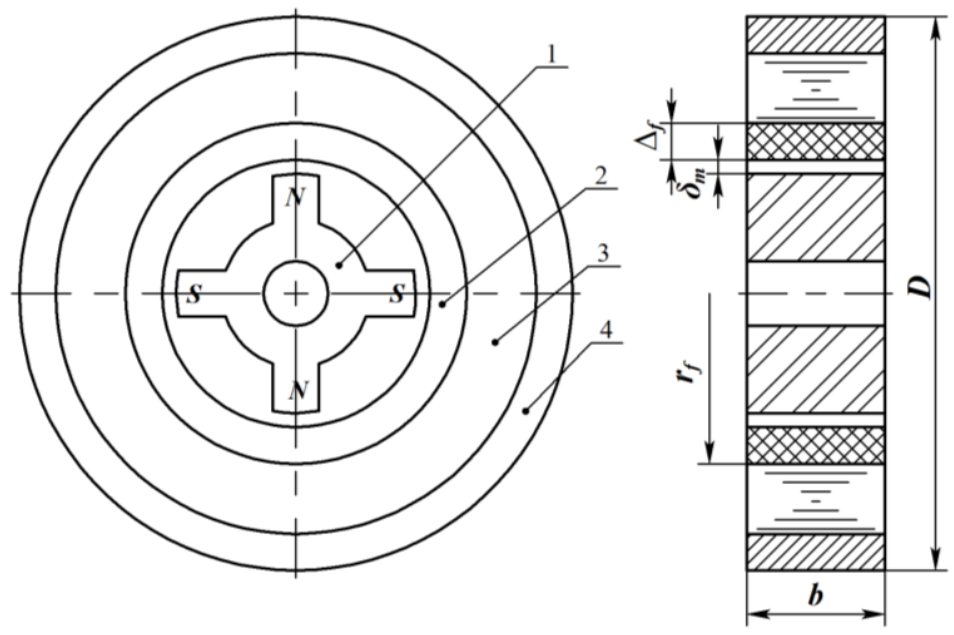

Fig. 1. The scheme of the tape winding torque motor:

1 - Permanent magnets (PM); 2 - Tube frame; 3 - Tape winding; 4 - Core.

The aim of this paper is the determination of the torque dependence over the design parameters of the motor, primarily geometric.

\section{The motor's torque}

It was previously shown [5] that the tape winding motor torque is given by the expression:

$$
T=\frac{\Delta^{2}}{2} \cdot \frac{U}{R} \cdot B(\delta)\left[N+\frac{2 r_{f}}{\Delta}-1\right] \cdot \frac{D\left(x, y, I_{0}\right)}{I_{0}},
$$

where $\Delta$ is the tape thickness; $U$ is the power supply voltage; $R$ is the plate resistance; $B(\delta)$ is magnetic flux density created by one pole; $N$ is the number of winding turns; $r_{f}$ is the tube frame radius; $D\left(x, y, I_{0}\right)$ is a double integral over the surface of the magnetic flux action.

In the above expression it is assumed that the thickness of the electrical insulation is small and neglected. Also, the expression (1) does not reflect the armature reaction on magnetic flux density. In addition, the component $\frac{2 r_{f}}{\Delta} \gg>1$ and the expression (1) can be reduced to the form:

$$
T=\frac{U}{R} \cdot\left(\frac{N \cdot \Delta}{2}+r_{f}\right) \cdot\left[\frac{B(\delta)}{I_{0}} \cdot \Delta \cdot D\left(x, y, I_{0}\right)\right] .
$$

Note that the expression in the square brackets represents the force produced by a single plate with 1 A current flowing through it:

$$
F_{01}=\frac{B(\delta)}{I_{0}} \cdot \Delta \cdot D\left(x, y, I_{0}\right) .
$$


The expression $\left(\frac{N \cdot \Delta}{2}+r_{f}\right)$ represents the medium radius $r_{m}$ of the tape winding. This allows us to indicate that:

$$
T=\frac{U}{R} \cdot r_{m} \cdot F_{01}
$$

\section{Results and discussion}

However it is necessary to take into account the insulation on the tape surface, which can be done with the copper space factor:

$$
k_{c}=\frac{\Delta}{\Delta_{i n}}
$$

where $\Delta_{i n}$ is the tape thickness with the insulation.

Then the expression for the medium radius will be presented as:

$$
r_{m}=\frac{N \cdot \Delta}{2 k_{c}}+r_{0}
$$

The flux density $B(\delta)$ and the integral $D\left(x, y, I_{0}\right)$ will also depend on the motor geometric parameters. The flux density mainly depends on the value of the non-magnetic gap $\delta_{n m}$, which is determined by the sum of the mechanical gap $\delta_{m}$, the thickness of the tube frame for winding the tape $\Delta_{f}$ and the tape winding thickness:

$$
\delta_{n m}=\delta_{m}+\Delta_{f}+\frac{\Delta \cdot N}{k_{c}} .
$$

Besides, as shown previously [6-8], the magnetic flux density also depends on the pole pairs number of the magnetic system. Also in [6] it is indicated that for a given tape width the value of the double integral $D\left(x, y, I_{0}\right)$ remains practically constant when the pairs of poles number of the magnetic system changes.

As a result, it is possible to estimate the influence of the force value $F_{01}$ at the motor's torque only by analyzing the particular motor scheme.

Let us consider the influence of the single plate resistance on the motor's torque. Resistance depends on the motor geometric parameters. As the poles number of the magnetic system increases, the plate length corresponding to the pole division will decrease. The plate resistance over its length will vary ambiguously. Numerical simulations have shown that the resistance of a single plate depends on the ratio of its length $a$ and width $b$. Varying this ratio $\beta=a / b$, when $b=$ const, a certain minimum resistance value is observed (Figure 2). During the modeling in the Electric Currents module of COMSOL, a copper plate with thickness $\Delta=10^{-4} \mathrm{~m}$, a width $\mathrm{b}=0.05 \mathrm{~m}$ was constructed. The plate length $\mathrm{a}_{\mathrm{i}}$ varied from the initial value $\mathrm{a}_{1}=0.135 \mathrm{~m}$. 


\section{$R \cdot 10^{-4}, \Omega$}

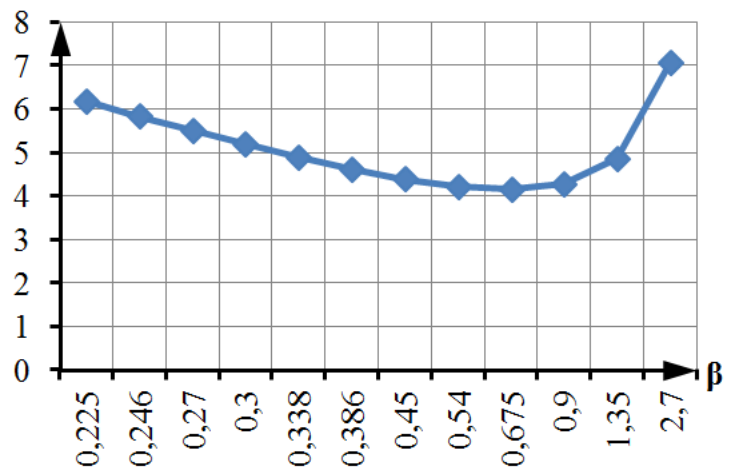

Fig. 2. The single plate resistance over ratio $\beta=a / b$.

The average plate length (pole pitch) is defined as

$$
a=\pi \cdot \frac{r_{m}}{p}
$$

where $p$ is a number of magnetic system pole-pairs.

The tape width is almost equal to the motor axial length $b$. The minimum plate resistance $R_{\min }$ is reached at a specific value of the ratio $\beta$. If we substitute (8) in the formula for the ratio $\beta$, we obtain:

$$
\beta=\frac{\pi}{p} \cdot \frac{r_{m}}{b}
$$

The necessary value of $\beta$ can be obtained by:

1. Changing the medium radius of the tape winding due to the number of winding turns selection $N$;

2. Selecting the pairs of poles number $p$;

3. Changing the tape width $b$.

The most rational approach is the third, since the first two require separate studies.

\section{Conclusion}

Developing the torque motor with the high specific characteristics it is necessary to consider the influence of the design parameters of both the magnetic circuit and windings. Firstly the poles pairs number that provides the maximum flux density in the gap should be determined. The plate length $a$ is chosen equal to the pole pitch of the magnetic circuit. The minimum value of $\beta$ when the plate resistance is also minimum is selected by changing the tape width. Then the plate resistance value is calculated and, according to the permitted winding current, the required number of turns are founded.

\section{References}

1. G. Dubrovskiy, A. Mikerov, V. Dzhankhotov and J. Pyrhonen, EPE-ECCE Europe 6910754 (2014) 
2. D. Hanselman, Brushless Permanent Magnet Motor Design. Second Edition (Magna Physics Publishing, United States of America, 2006)

3. A. Hughes and B. Drury, Electric Motors and Drives. Fundamentals, Types and Applications. 4th Edition (Elsevier-Newnes, Oxford, 2013)

4. P. Madaan, Brushless DC Motors. Part 1. Construction and Operating Principles (Cypress Semiconductor, India, 2013)

5. A. Dolgih (Ivanova), V. Martemyanov, SIBCON, 7491844 (2016) DOI: 10.1109/SIBCON.2016.7491844

6. A. Dolgih (Ivanova), V. Martemyanov, V. Borikov, Matec Web of Conf. 113, 01013 (2017) DOI: 10.1051/matecconf/201711301013

7. N. Natalinova, N. Ilina, E. Frantcuzskaia, IOP Conf. Ser.: Materials Science and Engineering 132(1), 012029 (2016) DOI: 10.1088/1757-899X/132/1/012029

8. I. A. Lezhnina, A. A. Boyakhchyan, K. V. Overchuk, A. A. Uvarov, Journal of Physics: Conf. Ser. 881(1), 012041 (2017) DOI: 10.1088/1742-6596/881/1/012041 